EPJ Web of Conferences 45, 01090 (2013)

DOI: $10.1051 /$ epjconf/20134501090

C Owned by the authors, published by EDP Sciences, 2013

\title{
Numerical experiments for turbulent flows
}

\author{
Jiří Trefilík ${ }^{1,2, a}$, Karel Kozel ${ }^{1,2, b}$, and Jaromír Příhoda ${ }^{2, c}$ \\ 1 Institute of Thermomechanics AS CR, v. v. i. \\ 2 Faculty of Mechanical Engineering, CVUT Prague
}

\begin{abstract}
The aim of the work is to explore the possibilities of modelling transonic flows in the internal and external aerodynamics. Several configurations were analyzed and calculations were performed using both inviscid and viscous models of flow. Viscous turbulent flows have been simulated using either zero equation algebraic Baldwin-Lomax model and two equation $k-\omega$ model in its basic version and improved TNT variant. The numerical solution was obtained using Lax-Wendroff scheme in the MacCormack form on structured non-ortogonal grids. Artificial dissipation was added to improve the numerical stability. Achieved results are compared with experimental data.
\end{abstract}

\section{Mathematical models}

\subsection{Navier-Stokes equations}

The two-dimensional laminar flow of a viscous compressible liquid is described by the system of Navier-Stokes equations

$$
W_{t}+F_{x}+G_{y}=R_{x}+S_{y}
$$

where

$$
W=\left(\begin{array}{c}
\rho \\
\rho u \\
\rho v \\
e
\end{array}\right), F=\left(\begin{array}{c}
\rho u \\
\rho u^{2}+p \\
\rho u v \\
(e+p) u
\end{array}\right), G=\left(\begin{array}{c}
\rho v \\
\rho u v \\
\rho v^{2}+p \\
(e+p) v
\end{array}\right)
$$

and

$$
R=\left(\begin{array}{c}
0 \\
\tau_{x x} \\
\tau_{x y} \\
u \tau_{x x}+v \tau_{x y}+\lambda T_{x}
\end{array}\right), S=\left(\begin{array}{c}
0 \\
\tau_{x y} \\
\tau_{y y} \\
u \tau_{x y}+v \tau_{y y}+\lambda T_{y}
\end{array}\right)
$$

with shear stresses given for the laminar flow by equations

$$
\tau_{x x}=\frac{2}{3} \eta\left(2 u_{x}-v_{y}\right), \tau_{x y}=\eta\left(u_{y}+v_{x}\right), \tau_{y y}=\frac{2}{3} \eta\left(-u_{x}+2 v_{y}\right) .
$$

This system is enclosed by the equation of state

$$
p=(\kappa-1)\left[e-\frac{1}{2} \rho\left(u^{2}+v^{2}\right)\right] \text {. }
$$

In the above given equations, $\rho$ denotes density, $u, v$ are components of velocity in the direction of axis $x, y, p$ is pressure, $e$ is total energy per a unit volume, $T$ is temperature, $\eta$ is dynamical viscosity and $\lambda$ is thermal conductivity coefficient. The parameter $\kappa=1.4$ is the adiabatic exponent.

\footnotetext{
a e-mail: jiri.trefilik@fs.cvut.cz

b e-mail: karel.kozel@fs.cvut.cz

c e-mail: prihoda@it.cas.cz
}

\subsection{Reynolds averaged Navier-Stokes equations}

For the modelling of a turbulent flow, the system of RANS (Reynolds Averaged Navier-Stokes) equations enclosed by a turbulence model is used. Two different turbulence models with the turbulent viscosity were tested, one algebraic, Baldwin-Lomax and the two-equation $k-\omega$ model according to Wilcox. The system of averaged Navier-Stokes equations is formally the same as (1), but this time the flow parameters represent only mean values in the Favre sense, see [3]. The shear stresses are given for the turbulent flows by equations

$$
\begin{aligned}
\tau_{x x} & =\frac{2}{3}\left(\eta+\eta_{t}\right)\left(2 u_{x}-v_{y}\right), \\
\tau_{x y} & =\left(\eta+\eta_{t}\right)\left(u_{y}+v_{x}\right), \\
\tau_{y y} & =\frac{2}{3}\left(\eta+\eta_{t}\right)\left(-u_{x}+2 v_{y}\right),
\end{aligned}
$$

where $\eta_{t}$ denotes the turbulent dynamic viscosity according to the Boussinesq hypothesis. The Reynolds number is defined by $R e=\frac{u_{\infty} L}{\eta_{\infty}}$ and the Mach number by $M=\sqrt{\frac{q}{a}}$ where $q=\sqrt{\left(u^{2}+v^{2}\right)}$ and $a$ is the local speed of sound.

All the computations were carried out using dimensionless variables with reference variables given by inflow values. The reference length $L$ is given by the width of the computational domain.

\section{Turbulence models}

\subsection{Baldwin-Lomax model}

Algebraic models are based on the model proposed for the boundary-layer flows by Cebeci and Smith. BaldwinLomax model is its modification applicable for general turbulent shear flows. The boundary layer is divided into two regions. In the inner (nearest to the wall) part, the turbulent viscosity is given by

$$
\eta_{t}=\rho F_{D}^{2} \kappa^{2} y^{2}|\Omega|,
$$


where $\Omega$ is the vorticity, which is in the $2 \mathrm{D}$ flow determined by

$$
\Omega=\frac{\partial u}{\partial y}-\frac{\partial v}{\partial x}
$$

and

$$
F_{D}=1-\exp \left(-\frac{y^{+}}{A^{+}}\right) .
$$

$y^{+}=\frac{u_{*} y}{v}$ denotes dimensionless distance from the wall, $v=$ $\frac{\mu}{\rho}$ is kinematic viscosity, $u_{*}=\sqrt{\left(\frac{\tau_{w}}{\rho}\right)}$ is so called friction velocity and $\tau_{w}=\mu\left(\frac{\partial u}{\partial y}\right)_{y=0}$.

The turbulent viscosity in the outer region is given by

$$
\eta_{t o}=\alpha \rho C_{c p} F_{w} F_{k},
$$

where $C_{c p}$ is a constant. Function $F_{w}$ is determined by the relation

$$
F_{w}=y_{\max } F_{\text {max }}
$$

for $F_{w}$ being the maximum of the function

$$
F=y F_{D}|\Omega|
$$

and $y_{\max }$ the distance from the wall in which $F\left(y_{\max }\right)=$ $F_{\text {max }}$ holds and

$$
F_{k}=\left[1+5.5\left(C_{K L} \frac{y}{y_{\max }}\right)^{6}\right]^{-1}
$$

The Baldwin-Lomax model (1978) contains following values of the constants: $\kappa=0.4, A^{+}=26, \alpha=0.0168$, $C_{c p}=1.6, C_{K L}=0.3$.

\section{$2.2 k-\omega$ model}

Two-equation models are based on transport equations for two characteristic scales of turbulent motion, mostly for the turbulent energy $k$ and dissipation rate $\epsilon$, often used in the form of specific dissipation rate $\omega=\epsilon / k$. These characteristics are computed from transport equations. Turbulent viscosity is defined as

$$
\eta_{t}=\rho \frac{k}{\omega}
$$

The standard Wilcox $k-\omega$ model is formed by the equations

$$
\frac{\partial}{\partial t}(\rho k)+\frac{\partial}{\partial x_{j}}\left(\rho u_{j} k\right)=P_{k}+\frac{\partial}{\partial x_{j}}\left[\left(\eta+\sigma^{*} \eta_{t}\right) \frac{\partial k}{\partial x_{j}}\right]-\beta^{*} \rho k \omega,
$$

$$
\frac{\partial}{\partial t}(\rho \omega)+\frac{\partial}{\partial x_{j}}\left(\rho u_{j} \omega\right)=\gamma \frac{\omega}{k} P_{k}+\frac{\partial}{\partial x_{j}}\left[\left(\eta+\sigma \eta_{t}\right) \frac{\partial \omega}{\partial x_{j}}\right]-\beta \rho \omega^{2},
$$

where $P_{k}=\tau_{i j} \partial u_{i} / \partial x_{j}$ represents the production of turbulent energy. Model coefficients are given by values: $\alpha=5 / 9, \beta=3 / 40, \beta^{*}=9 / 100, \sigma=1 / 2$ and $\sigma^{*}=1 / 2$, $i, j \in\{1,2\}$.

So called TNT modification of k- $\omega$ model:

$$
\frac{\partial(\rho k)}{\partial t}+\frac{\partial\left(\rho u_{j} k\right)}{\partial x_{j}}=P_{k}+\frac{\partial}{\partial x_{j}}\left[\left(\mu+\sigma_{k} \mu_{t}\right) \frac{\partial k}{\partial x_{j}}\right]-\beta^{*} \rho k \omega,
$$

$\frac{\partial(\rho \omega)}{\partial t}+\frac{\partial\left(\rho u_{j} \omega\right)}{\partial x_{j}}=\alpha \frac{\omega}{k} P_{k}+\frac{\partial}{\partial x_{j}}\left[\left(\mu+\sigma_{\omega} \mu_{t}\right) \frac{\partial \omega}{\partial x_{j}}\right]-\beta \rho k \omega^{2}+C_{D}$,

where

$$
C_{D}=\sigma_{d} \frac{\rho}{\omega} \max \left(\frac{\partial k}{\partial x_{i}} \frac{\partial \omega}{\partial x_{i}}, 0\right)
$$

and $\alpha_{\omega}=\frac{\beta}{\beta_{*}}-\frac{\sigma_{\omega} \kappa^{2}}{\sqrt{\beta^{*}}}, \kappa=0.41, \beta=\frac{3}{40}, \beta^{*}=\frac{9}{100}$, $\sigma_{\omega}=0.5, \sigma_{k}=0.666$.

\section{Numerical methods}

For the modelling of the flow cases Lax-Wendroff finite volume method scheme was used on non-orthogonal structured grids of quadrilateral and hexahedral cells $D_{i j(k)}$.

- Predictor step:

$$
W_{i, j}^{n+1 / 2}=W_{i, j}^{n}-\frac{\Delta t}{\mu_{i, j}} \sum_{k=1}^{4}\left[\left(\tilde{F}_{k}^{n}-\frac{1}{R e} R_{k}^{n}\right) \Delta y_{k}-\left(\tilde{G}_{k}^{n}-\frac{1}{R e} S_{k}^{n}\right) \Delta x_{k}\right] .
$$

- Corrector step:

$$
\begin{aligned}
W_{i, j}^{n+1}= & \frac{1}{2}\left(W_{i, j}^{n}+W_{i, j}^{n+1 / 2}\right)-\frac{\Delta t}{2 \mu_{i, j}} \sum_{k=1}^{4}\left[\left(\tilde{F}_{k}^{n+1 / 2}-\frac{1}{R e} R_{k}^{n+1 / 2}\right) \Delta y_{k}\right. \\
& \left.-\left(\tilde{G}_{k}^{n+1 / 2}-\frac{1}{R e} S_{k}^{n+1 / 2}\right) \Delta x_{k}\right]+A D\left(W_{i, j}^{n}\right) .
\end{aligned}
$$

The Mac Cormack scheme in the cell centered form was applied to solving the system of RANS equations. Convective terms $F, G$ are considered in predictor step in forward form and in the corrector step in upwind form of the first order of accuracy, dissipative terms in central form of the second order of accuracy. To indicate this we denote their numerical approximation as $\tilde{F}, \tilde{G}$.

The value $\mu_{i j(k)}$ represents the surface (volume) of the cell. Figure 14 shows evaluation of derivatives on edges of cells: We imagine a virtual cell as shown in the picture. We know the values in the centers of the cells and we define the other two as a mean value of its surrounding cells. From that we extrapolate to its edges and then we apply Green's formula.

The scheme was extended to include Jameson's artificial dissipation because of the stability of the method

$$
\begin{aligned}
A D\left(W_{i, j}^{n}\right) & =C_{1} \psi_{1}\left(W_{i-1, j}^{n}-2 W_{i, j}^{n}+W_{i+1, j}^{n}\right) \\
& +C_{2} \psi_{2}\left(W_{i, j-1}^{n}-2 W_{i, j}^{n}+W_{i, j+1}^{n}\right),
\end{aligned}
$$

where

$$
\begin{aligned}
& \psi_{1}=\frac{\left|p_{i-1, j}^{n}-2 p_{i, j}^{n}+p_{i+1, j}^{n}\right|}{\left|p_{i-1, j}^{n}\right|+\left|p_{i, j}^{n}\right|+\left|p_{i+1, j}^{n}\right|}, \\
& \psi_{2}=\frac{\left|p_{i, j-1}^{n}-2 p_{i, j}^{n}+p_{i, j+1}^{n}\right|}{\left|p_{i, j-1}^{n}\right|+\left|p_{i, j}^{n}\right|+\left|p_{i, j+1}^{n}\right|} .
\end{aligned}
$$


Inlet boundary conditions IBC $\mathcal{A}$ were used for inviscid compressible flows; IBC $\mathcal{B}$ were used for inviscid compressible flows. A nonzero angle of attack $\alpha_{1}$ was used only in the case of flows through DCA cascade.

On the outlet we prescribed only pressure $p_{2}=p_{1}$ and the other values were extrapolated from the flow field.

Further on there are three other types of boundary conditions: solid wall, symmetry axis and periodicity. These conditions are implemented by using virtual cells. Such cells adjoin from outside on the boundary cells and we prescribe values of unknowns inside of them to obtain the desired effect.

Solid wall (an inviscid flow): velocity components prescribed so that the sum of velocity vectors equals to zero in its tangential component. Solid wall (a viscous flow): velocity components were prescribed so that the sum of velocity vectors equals zero. In both cases the rest of unknowns is the same in both the virtual and the boundary cell.

Symmetry axis: this condition was realized by the same way as the wall condition for an inviscid flow.

Periodicity condition: taking two corresponding segments of boundary we prescribe into virtual cells of the first segment the values of unknowns contained in the boundary cells of the second and vice-versa.

Initial conditions were prescribed to comply with the inlet conditions.

\section{Results}

The following pictures ilustrate results obtained during the calculations. First three of them present simulation of the flow through the DCA cascade with the inlet Mach number $M_{\infty}=0.832$ and angle of attack $\alpha=0^{\circ}$. Result obtained by employing TNT scheme shows very good agreement with experiment. Then similar configuration with inlet Mach number $M_{\infty}=0.863$ and angle of attack $\alpha=0^{\circ}$ follows. In this case for the modelling of turbulence the Baldwin-Lomax model was used. The last configuration of this type is for inlet Mach number $M_{\infty}=0.982$ with TNT model and again there is a good agreement. Second type domain DEIW calculations follow. First there is an inviscid flow in the same configuration as the reference result complemented by the viscous flow computation on a symmetrical domain. But the value of Reynolds number is 10 times bigger, which might be the of cause why the separation is more distinct.

\section{Acknowledgment}

The work was partly supported by by the institutional support RVO 61388998 and by the grant projects GA AS CR IAA 200760 81, GA CR P101/10/1329, P 101/12/1271 and SGS 10/243/OHK2/3T/12.

\section{References}

1. R. Dvořák, Transonic Flows, Academia, Prague (1986, in Czech).

2. R. Dvořák, K. Kozel, Mathematical modelling in aerodynamics, CTU in Prague, Prague (1996, in Czech)
3. A. Favre, Equations des gaz turbulents compressibles, Jour. de Mecanique, 4, 361-390 (1965)

4. M. Feistauer, J. Felcman, I. Straškraba, Mathematical and Computational Methods for Compressible Flow, Oxford University Press (2003)

5. C. Hirsch, Numerical Computation of Internal and External Flows, Volume II, - Computational Methods for Inviscid and Viscous Flows, John Willey\&Sons (1990)

6. J. Holman, J. Furst, Proceedings: Colloquium Fluid Dynamics 2008, 11-12, Institute of Thermomechanics, AS CR, v. v. i., Prague (2008)

7. J. Huml, J. Furst, K. Kozel, J. Př́ihoda, Proceedings: Topical Problems of Fluid Dynamics 2009, 45-48, Institute of Thermodynamics, AS CR, v. v. i., Prague (2009, in Czech).

8. J. Huml, J. Holman, J. Furst, K. Kozel, Proceedings: Topical Problems of Fluid Dynamics 2010, 73-76, Institute of Thermomechanics, AS CR, v. v. i., Prague (2010)

9. P. Pořízková, Numerical Solution of Compressible Flows Using Finite Volume Method, PhD Dissertation CTU in Prague, Faculty of Mechanical Engineering, CTU in Prague, Prague (2009, in Czech).

10. J. Př́ihoda, P. Louda, Mathematical modelling of turbulent flow, CTU in Prague, Prague 2007 (in Czech)

11. J. Šimonek, K. Kozel, J. Trefilík, Proceedings: Topical Problems of Fluid Mechanics 2007, 169-172, Institute of Thermomechanics, AS CR, v. v. i., Prague (2007) 


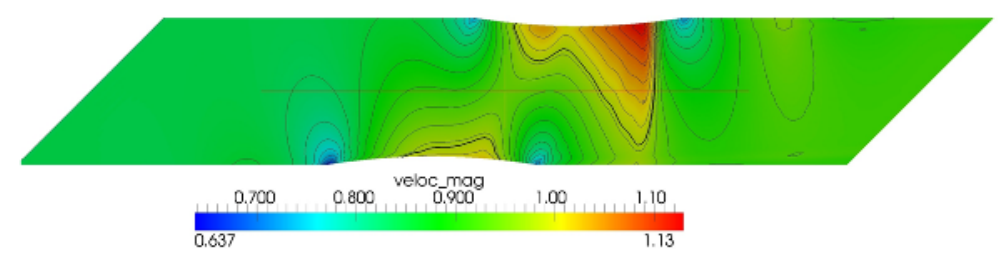

Fig. 3. DCA cascade, inviscid flow, $M_{\infty}=0.88, \alpha=0.6^{\circ}$, Mach number isolines

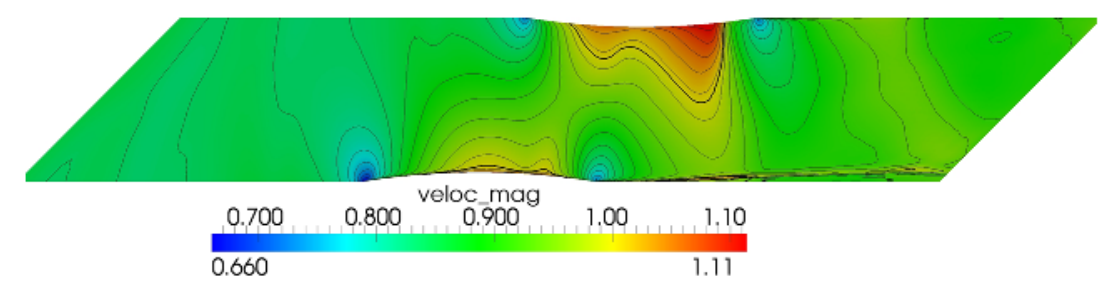

Fig. 4. DCA cascade, viscous flow, $M_{\infty}=0.88, \alpha=0.6^{\circ}, R e=10^{7}, k-\omega$ model, Mach number isolines

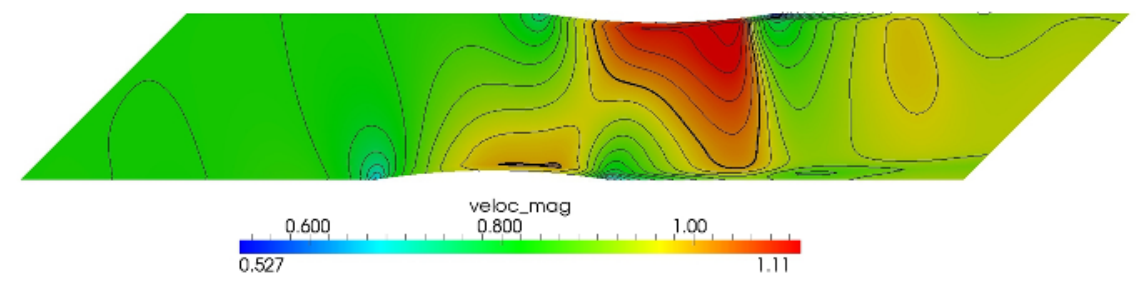

Fig. 5. DCA cascade, viscous flow, $M_{\infty}=0.89, \alpha=0.5^{\circ}, R e=10^{7}, k-\omega$ model (TNT variant), Mach number isolines

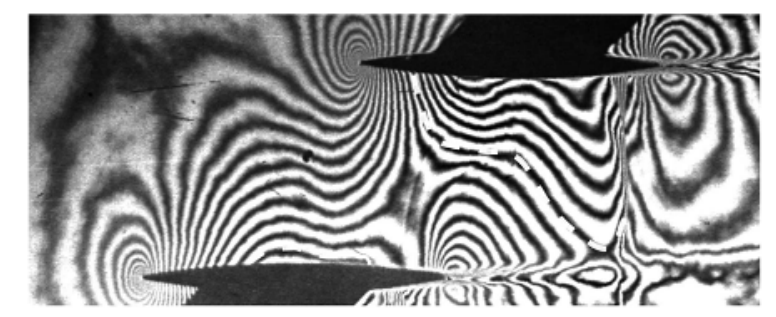

Fig. 6. Experimental data, $M_{\infty}=0.832, \alpha=0^{\circ}$

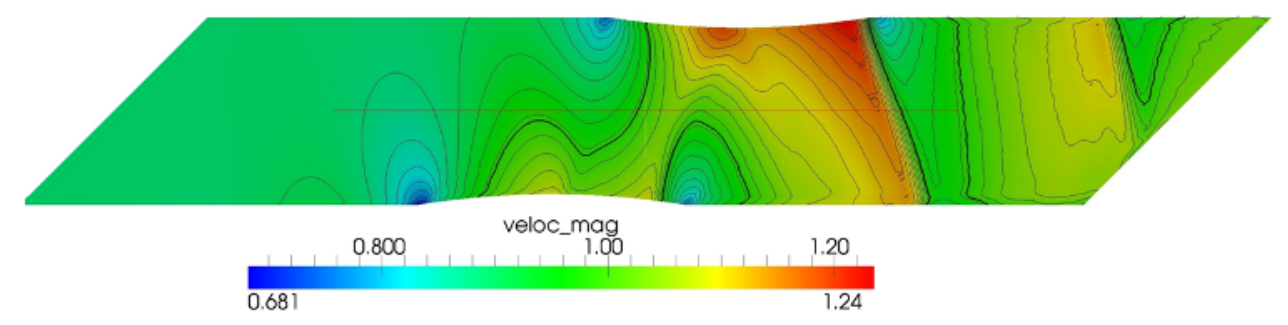

Fig. 7. DCA cascade, inviscid flow, $M_{\infty}=0.98, \alpha=3.0^{\circ}$, Mach number isolines

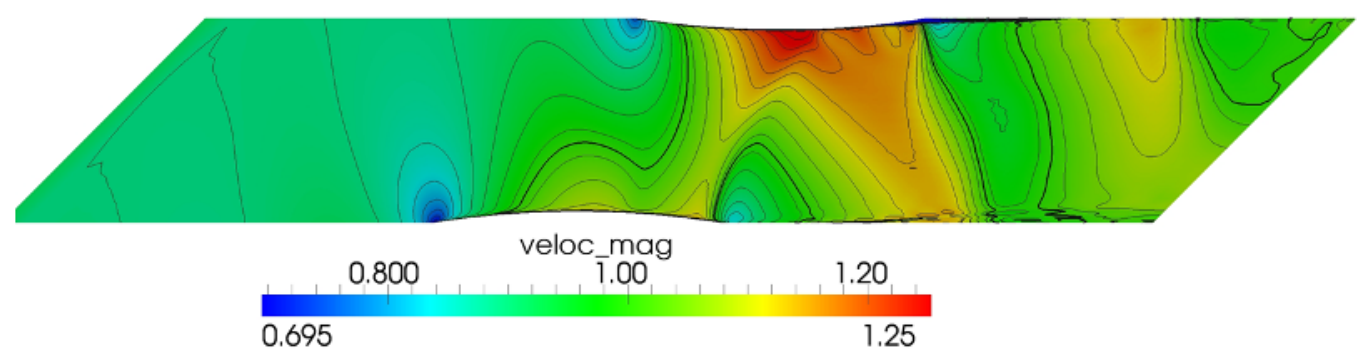

Fig. 8. DCA cascade, viscous flow, $M_{\infty}=0.98, \alpha=3.0^{\circ}, R e=10^{7}$, Baldwin-Lomax model, Mach number isolines 


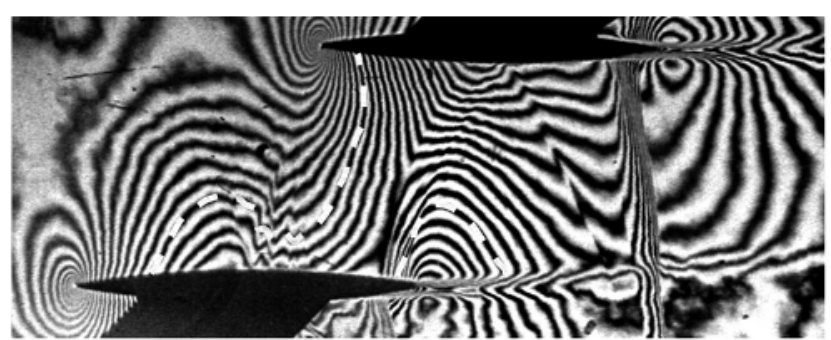

Fig. 9. Experimental data, $M_{\infty}=0.863, \alpha=0^{\circ}$

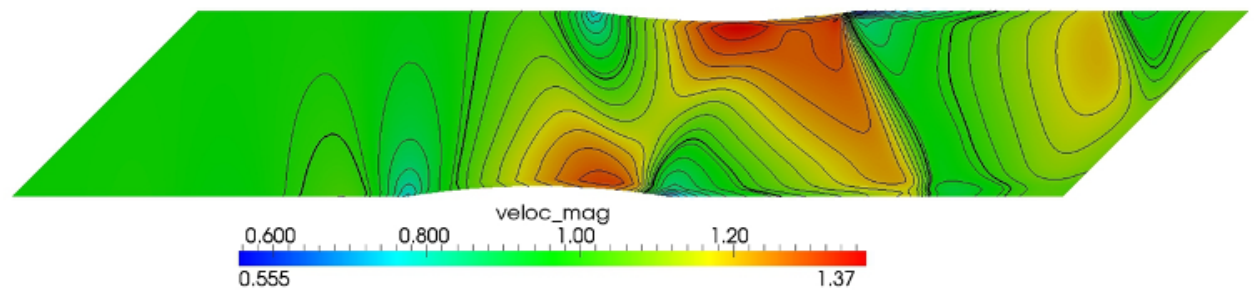

Fig. 10. DCA cascade, viscous flow, $M_{\infty}=1.07, \alpha=2.3^{\circ}, R e=10^{7}, k-\omega$ model (TNT variant), Mach number isolines

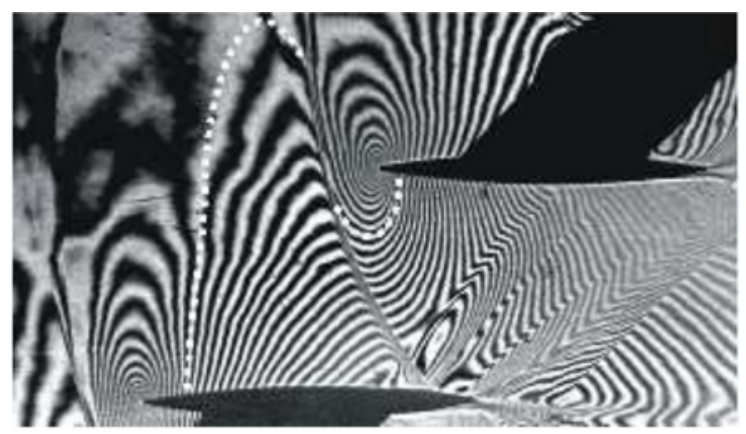

Fig. 11. Experimental data, $M_{\infty}=0.982, \alpha=0^{\circ}$

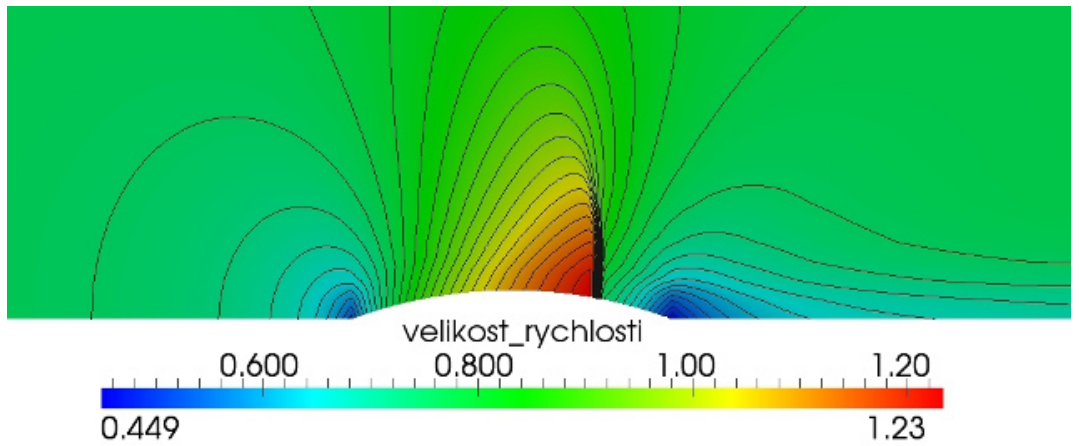

Fig. 12. DEIW configuration, inviscid flow, $M_{\infty}=0.775, \alpha=0^{\circ}$, Mach number isolines 


\section{EPJ Web of Conferences}

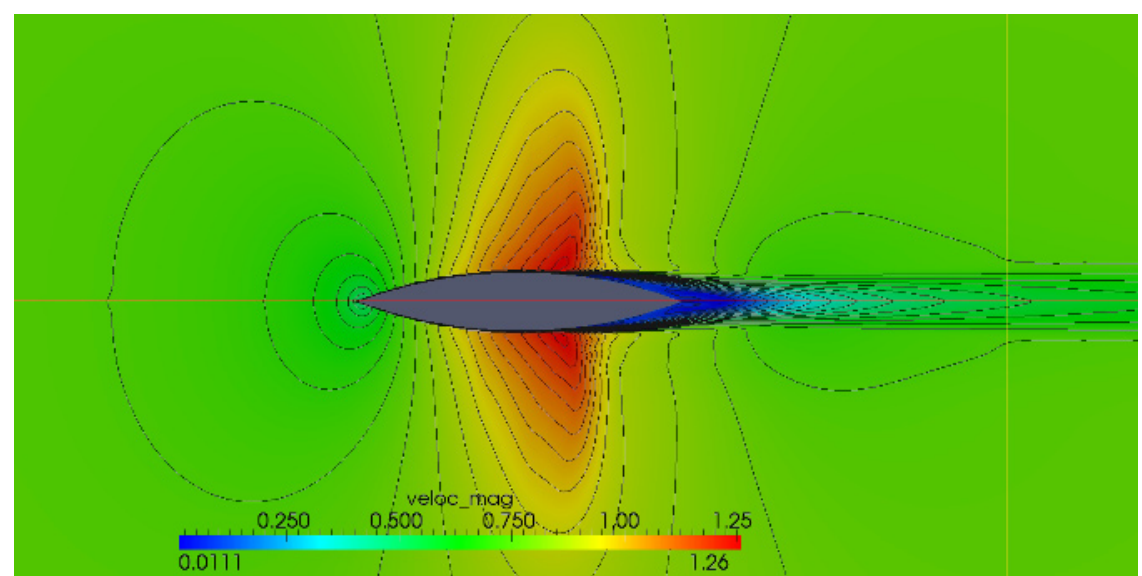

Fig. 13. symmetric DEIW configuration, viscous flow, $M_{\infty}=0.775, \alpha=0^{\circ}, R e=10^{6}, k-\omega$ model (TNT variant), Mach number isolines

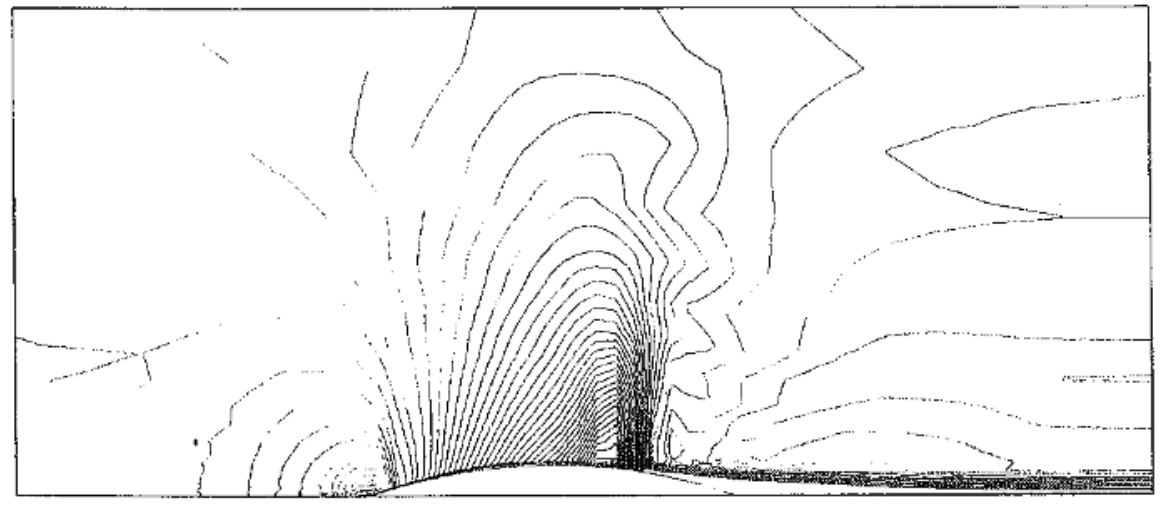

Fig. 14. DEIW configuration reference result, viscous flow, $M_{\infty}=0.775, \alpha=0^{\circ}, R e=10^{7}$, simple mixing length model, Mach number isolines 